Dalton Euclides Sarango-Guamán; Diego Armando Masaquiza-Moposita; Junior Stalin Vargas-Hidalgo

http://dx.doi.org/10.35381/r.k.v6i11.1118

\title{
Validación in vivo de la Metodología Producción de Gas in vitro en fermentadores Post Gástricos
}

\section{In vivo validation of the in vitro Gas Production Methodology in Post Gastric Fermenters}

Dalton Euclides Sarango-Guamán

daltonsarango@yahoo.com

Escuela Superior Politécnica de Chimborazo, Riobamba

Ecuador.

https://orcid.org/0000-0002-2890-0882

Diego Armando Masaquiza-Moposita

diegomasaquiza@gmail.com

Escuela Superior Politécnica de Chimborazo, Riobamba

Ecuador.

https://orcid.org/0000-0001-5176-8261

Junior Stalin Vargas-Hidalgo

junior.vargas@espoch.edu.ec

Escuela Superior Politécnica de Chimborazo, Riobamba

Ecuador.

https://orcid.org/0000-0003-1245-7609

Recepción: 12 de julio 2020

Revisado: 30 de agosto 2020

Aprobación: 20 de diciembre 2020

Publicación: 01 de enero 2021 
Revista Arbitrada Interdisciplinaria KOINONIA

Año VI. Vol VI. N${ }^{\circ} 1$. Enero - Junio. 2021

Hecho el depósito de Ley: FA2016000010 ISSN: 2542-3088

FUNDACIÓN KOINONIA (F.K). Santa Ana de Coro. Venezuela.

Dalton Euclides Sarango-Guamán; Diego Armando Masaquiza-Moposita; Junior Stalin Vargas-Hidalgo

\title{
RESUMEN
}

El trabajo tuvo como finalidad validar la metodología in vitro de producción de gas con datos de digestibilidad in vivo en conejos. Se utilizaron datos obtenidos de ensayos in vitro e in vivo realizados en el departamento de producción animal de la Universidad Politécnica de Madrid, se correlacionaron para validar los mejores tiempos e índices de la curva modelizada de producción de gas in vitro, con los datos de digestibilidad in vivo. Las correlaciones más altas se obtuvieron entre desaparición de la fibra soluble a nivel ileal y el volumen de gas obtenido a 6 horas de fermentación; así mismo entre la degradación de la fibra neutro detergente in vivo y el volumen de producción de gas desde las 29 a 96 horas de fermentación. La metodología de producción de gas es una buena alternativa para estudiar la degradación de la fibra, sus fracciones, en el tracto digestivo de conejos.

Descriptores: Análisis cuantitativo; genética animal; nutrición animal. (Palabras tomadas del Tesauro UNESCO).

\begin{abstract}
The purpose of the work was to validate the in vitro gas production methodology with in vivo digestibility data in rabbits. Data obtained from in vitro and in vivo tests carried out in the department of animal production of the Polytechnic University of Madrid were used, they were correlated to validate the best times and indices of the modeled curve of in vitro gas production, with the digestibility data in vivo. The highest correlations were obtained between the disappearance of soluble fiber at the ileal level and the volume of gas obtained at 6 hours of fermentation; likewise between the degradation of the neutral detergent fiber in vivo and the volume of gas production from 29 to 96 hours of fermentation. The gas production methodology is a good alternative to study the degradation of fiber, its fractions, in the digestive tract of rabbits.
\end{abstract}

Descriptors: Quantitative analysis; animal genetics; animal nutrition. (Words taken from the UNESCO Thesaurus). 


\section{INTRODUCCIÓN}

La digestibilidad es el resultado de varios procesos que incluyen hidrolisis, absorción, secreción y pasaje de nutrientes del alimento al ser vivo (Wilfart et al., 2008). E conocimiento de la metodología para la determinación de la digestibilidad de los nutrientes representa el pilar básico de la nutrición en la producción animal y un interés puntual en la industria de los alimentos para balanceados y materias primas. Las pruebas comúnmente utilizadas se basan en un balance de ingesta/excreta que se realizan con experimentaciones denominados in vivo. Estas investigaciones a más de ser prolongadas están sujetas al tiempo y consumo de alimento (De Blas \& Wiseman, 2010). En el caso en fermentadores postgástricos, los valores nutricionales de los alimentos se determinan considerando criterios tales como la digestibilidad ileal y fecal. Estas digestibilidades requieren del equipamiento de jaulas metabólicas y el sacrificio de los animales, lo que va en contra de las actuales normas de bioética que exigen el reemplazo de animales como modelos de investigación por metodologías de experimentación in vitro.

Desde hace muchas décadas pruebas de digestibilidad in vitro se vienen realizando en rumiantes. Los precursores (Tilley \& Terry, 1963) marcaron un hito en lo que a experimentación se refiere años más tarde se han ido perfeccionando técnicas de producción de gas en rumiantes (Menke et al., 1979) y desarrollando investigaciones en aves y porcinos (Williams et al., 1995; Kwakkel et al., 1997). En conejos su uso ha empezado a utilizarse, mostrando resultados prometedores (Dihigo, 2005). Ante estas perspectivas se planteó con el presente trabajo validar la metodología in vitro de producción de gas con datos de digestibilidad in vivo en conejos. 
Dalton Euclides Sarango-Guamán; Diego Armando Masaquiza-Moposita; Junior Stalin Vargas-Hidalgo

\section{MATERIALES Y MÉTODOS}

Los datos utilizados fueron obtenidos de ensayos in vitro e in vivo realizados en el departamento de producción animal de la Universidad Politécnica de Madrid.

\section{Diseño experimental}

Se utilizó correlaciones para validar los mejores tiempos e índices de la curva modelizada de producción de gas in vitro, con los datos de digestibilidad in vivo.

\section{Variables en estudio}

Las variables evaluadas fueron:

Mejor tiempo de producción de gas in vitro que se correlacionó con la digestibilidad in vivo

Mejor índice del modelo de producción de gas in vitro que se correlacionó con la digestibilidad in vivo.

\section{Análisis estadístico}

Para el análisis estadístico de los resultados se utilizaron correlaciones con el procedimiento PROC CORR del SAS (SAS Inst. Inc., Cary, NC). Además, se modelizaron los datos de producción de gas con un modelo logístico, utilizando el mismo programa estadístico.

\section{RESULTADOS}

En razón de la recopilación de los datos, se presentan los siguientes resultados:

Correlación de parámetros de producción de gas in vitro y digestibilidad ileal in vivo

En la tabla 1, se muestra los resultados de las correlaciones de diferentes parámetros de producción de gas in vitro y digestibilidad ileal in vivo de la materia seca y diferentes fracciones de fibra (fibra dietética total, fibra neutro detergente y fibra soluble). 


\section{Tabla 1.}

Correlación ( $r$ ) de diferentes parámetros de producción de gas in vitro y digestibilidad ileal in vivo de la materia seca y diferentes fracciones de fibra.

\begin{tabular}{lcccc}
\hline \multirow{2}{*}{ Parámetros } & \multicolumn{5}{l}{ Digestibilidad ileal in vivo } & \\
\cline { 2 - 5 } & $\begin{array}{l}\text { Materia } \\
\text { seca }\end{array}$ & $\begin{array}{l}\text { Fibra } \\
\text { total }\end{array}$ & $\begin{array}{l}\text { dietética } \\
\text { fibra } \\
\text { detergente }\end{array}$ & $\begin{array}{l}\text { neutro } \\
\text { Fibra } \\
\text { soluble }\end{array}$ \\
\hline Tiempo de fermentación & (horas) & & & \\
6 & $-0,470$ & $\mathbf{0 . 8 5}$ & 0,480 & $\mathbf{0 . 8 1}$ \\
11 & $-0,430$ & $\mathbf{0 . 8 4}$ & 0,590 & $\mathbf{0 . 7 3}$ \\
23 & $-0,420$ & $\mathbf{0 . 8 6}$ & 0,660 & $\mathbf{0 . 7 0}$ \\
29 & $-0,400$ & $\mathbf{0 . 8 8}$ & $\mathbf{0 . 7 2}$ & 0,690 \\
48 & $-0,330$ & $\mathbf{0 . 8 8}$ & $\mathbf{0 . 7 8}$ & 0,670 \\
55 & $-0,310$ & $\mathbf{0 . 8 8}$ & $\mathbf{0 . 8 0}$ & 0,660 \\
72 & $-0,300$ & $\mathbf{0 . 8 9}$ & $\mathbf{0 . 8 2}$ & 0,660 \\
96 & $-0,290$ & $\mathbf{0 . 8 9}$ & $\mathbf{0 . 8 3}$ & 0,660 \\
Parámetros de la curva de producción de gas & & \\
Volumen final & $-0,280$ & $\mathbf{0 . 8 9}$ & $\mathbf{0 . 8 5}$ & 0,640 \\
Constante & $-0,280$ & 0,690 & 0,220 & $\mathbf{0 . 7 0}$ \\
Tiempo de espera & 0,360 & $-0,500$ & 0,050 & $-0,680$ \\
\hline
\end{tabular}

Los valores en marcados negrita corresponden a datos con diferencia significativas $(\mathrm{P}<$ $0,05)$.

No se encontraron correlaciones $(P>0,05)$ entre la digestibilidad de materia seca ileal in vivo, con ninguno de los parámetros de producción de gas in vitro estudiados. Mientras que la degradabilidad de la fibra total o sus fracciones solubles o insolubles se encontró valores de correlación significativas con los parámetros in vitro estudiados.

Lo que corresponde a degradabilidad ileal in vivo de fibra dietética total tubo correlaciones más altas a las 72 y 96 horas de fermentación in vitro y con el volumen final de producción de gas $(P<0,05 ; r=0,89)$ mientras que la constante de producción de gas ( $\mathrm{ml} / \mathrm{hora}$ ), y el tiempo de espera para el inicio de la fermentación (horas) no se correlacionaron ( $\mathrm{P}>$ 0,$05 ; r=0,69$ y $r=-0,50$ respectivamente). En cuanto a la desaparición ileal de fibra 
neutro detergente se determinó que la fermentación a las 96 horas y el volumen final de producción de gas son los que mejor se correlacionaron a esta $(P<0,05 ; r=0,83$ y $r=0,85$ respectivamente). Finalmente, en lo que respecta a la desaparición de la fibra soluble a nivel ileal se determinó que la fermentación a las 6 horas y la constante de fermentación son las que mejor se correlaciona con este ( $P<0,05 ; r=0,86 ; r=0,70$ respectivamente).

\section{Correlación de parámetros de producción de gas in vitro y digestibilidad cecal in vivo.}

En la tabla 2, muestra los resultados de las correlaciones de diferentes parámetros de producción de gas in vitro y digestibilidad cecal in vivo de la materia seca y diferentes fracciones de fibra (fibra dietética total, fibra neutra detergente y fibra soluble). La digestibilidad cecal in vivo de la materia seca se correlaciono con el volumen de producción de gas producido a las $29,48,55,72$ y 96 horas, y con el volumen final de producción de gas $(P<0,05 ; r>0,70)$. Siendo el volumen final el que presento la mayor correlación $(r=0,87)$.

Tanto la desaparición de fibra dietética total y fibra neutro detergente no se correlacionaron $(P>0,05 ; r<0,40)$ con ninguno de los parámetros estudiados de la producción de gas in vitro. Mientras que la desaparición de la fibra soluble in vivo a nivel cecal se correlaciono negativamente con el volumen de producción de gas a las 6, 11 y 23 horas de producción de gas, y con la constante modelizada de la curva de producción de gas in vitro $(P<0,05 ; r<-0,72)$. Siendo la producción de gas a las 6 horas la que presento la correlación negativa más alta $(r=-0,83)$ 


\section{Tabla 2.}

Correlación ( $r$ ) de diferentes parámetros de producción de gas In Vitro y digestibilidad cecal In Vivo de la materia seca y diferentes fracciones de fibra.

\begin{tabular}{|c|c|c|c|c|}
\hline \multirow[b]{2}{*}{ Parámetros } & \multicolumn{4}{|c|}{ Digestibilidad cecal in vivo } \\
\hline & $\begin{array}{l}\text { Materia } \\
\text { seca }\end{array}$ & $\begin{array}{l}\text { Fibra dietética } \\
\text { total }\end{array}$ & $\begin{array}{ll}\text { Fibra } & \text { neutro } \\
\text { detergente } & \end{array}$ & $\begin{array}{l}\text { Fibra } \\
\text { soluble }\end{array}$ \\
\hline \multicolumn{5}{|c|}{ Tiempo de fermentación (horas) } \\
\hline 6 & 0,510 & 0,170 & 0,250 & -0.83 \\
\hline 11 & 0,590 & 0,260 & 0,250 & -0.75 \\
\hline 23 & 0,650 & 0,240 & 0,210 & -0.71 \\
\hline 29 & 0.70 & 0,240 & 0,200 & $-0,690$ \\
\hline 48 & 0.75 & 0,300 & 0,230 & $-0,660$ \\
\hline 55 & 0.78 & 0,320 & 0,250 & $-0,650$ \\
\hline 72 & 0.82 & 0,350 & 0,290 & $-0,640$ \\
\hline 96 & 0.85 & 0,380 & 0,320 & $-0,630$ \\
\hline \multicolumn{5}{|c|}{ Parámetros de la curva de producción de gas } \\
\hline Volumen final $(\mathrm{ml} / \mathrm{g})$ & 0.87 & 0,400 & 0,330 & $-0,620$ \\
\hline Constante (ml/hora) & 0,150 & $-0,180$ & 0,085 & -0.72 \\
\hline $\begin{array}{l}\text { Tiempo de espera } \\
\text { (horas) }\end{array}$ & $-0,008$ & 0,110 & $-0,097$ & 0,700 \\
\hline \multicolumn{5}{|c|}{$\begin{array}{l}\text { Los valores en marcados negrita corresponden a datos con diferencia significativas }(P< \\
0,05) \text {. }\end{array}$} \\
\hline \multicolumn{5}{|c|}{$\begin{array}{l}\text { Correlación de parámetros de producción de gas in vitro y digestibilidad fecal in } \\
\text { vivo. }\end{array}$} \\
\hline \multicolumn{5}{|c|}{$\begin{array}{l}\text { En la tabla } 3 \text {, se muestra los resultados de las correlaciones de diferentes parámetros de } \\
\text { producción de gas in vitro y digestibilidad fecal in vivo de la materia seca y diferentes } \\
\text { fracciones de fibra (fibra dietética total, fibra neutro detergente y fibra soluble). }\end{array}$} \\
\hline \multicolumn{5}{|c|}{$\begin{array}{l}\text { La digestibilidad de la materia seca y la desaparición de la fibra soluble a nivel fecal, no } \\
\text { se correlacionaron }(P>0,05) \text { con ninguno de los parámetros estudiados de la producción } \\
\text { de gas in vitro. }\end{array}$} \\
\hline
\end{tabular}


La degradabilidad de la fibra dietética total se correlaciono con todas las horas a las que se midió el volumen de producción de gas y con el volumen final de producción de gas $(P<0,05 ; r>0,81)$. Siendo el volumen final y el volumen de gas obtenido a las 96 de fermentación, el que mejor se correlaciono $(r=0,92)$. La desaparición de la fibra neutro detergente in vivo se correlaciono $(P<0,05 ; r>0,80)$ con el volumen de producción de gas a las 29, 48, 55, 72 y 96 horas de fermentación, y con el volumen final de producción de gas. Siendo con el volumen final de producción de gas con el que mejor correlación se obtuvo $(r=0,87)$.

Tabla 3.

Correlación (r) de diferentes parámetros de producción de gas In Vitro y digestibilidad fecal In Vivo de la materia seca y diferentes fracciones de fibra.

\begin{tabular}{|c|c|c|c|c|}
\hline \multirow[b]{2}{*}{ Parámetros } & \multicolumn{4}{|c|}{ Digestibilidad fecal in vivo } \\
\hline & $\begin{array}{l}\text { Materia } \\
\text { seca }\end{array}$ & $\begin{array}{l}\text { Fibra dietética } \\
\text { total }\end{array}$ & $\begin{array}{ll}\begin{array}{l}\text { Fibra } \\
\text { detergente }\end{array} & \text { neutro } \\
\end{array}$ & $\begin{array}{l}\text { Fibra } \\
\text { soluble }\end{array}$ \\
\hline \multicolumn{5}{|c|}{ Tiempo de fermentación (horas) } \\
\hline 6 & 0,190 & 0.81 & 0,520 & $-0,110$ \\
\hline 11 & 0,290 & 0.82 & 0,620 & $-0,082$ \\
\hline 23 & 0,360 & 0.84 & 0,660 & $-0,045$ \\
\hline 29 & 0,420 & 0.86 & 0.71 & $-0,011$ \\
\hline 48 & 0,500 & 0.88 & 0.77 & 0,043 \\
\hline 55 & 0,540 & 0.89 & 0.80 & 0,069 \\
\hline 72 & 0,590 & 0.91 & 0.83 & 0,100 \\
\hline 96 & 0,630 & 0.92 & 0.85 & 0,140 \\
\hline \multicolumn{5}{|c|}{ Parámetros de la curva de producción de gas } \\
\hline Volumen final & 0,650 & 0.92 & 0.87 & 0,130 \\
\hline Constante & $-0,032$ & 0,550 & 0,230 & $-0,098$ \\
\hline Tiempo de espera & 0,220 & $-0,400$ & 0,005 & 0,077 \\
\hline
\end{tabular}

Los valores en marcados negrita corresponden a datos con diferencia significativas $(\mathrm{P}<$ $0,05)$. 


\section{DISCUSIÓN}

La correlación de la desaparición de la fibra soluble a nivel ileal con las primeras horas de fermentación muestra que esta fracción es de rápida fermentación y podría estar producida por la rica microbiota que se encuentra a nivel ileal en los conejos (GómezConde, et al., 2007), o incluso por las enzimas de origen microbiano de las heces blandas consumidas durante la cecotrofia. (Marounek, et al., 2005), demostró una alta actividad peptinolitica en el tracto digestivo anterior (estómago e intestino delgado). En contraste la fibra neutro detergente se encuentra mejor correlacionado a últimas horas de fermentación, relacionándose esto con el mayor tiempo que requiere la fracción insoluble de la fibra para fermentarse. Esto corroboraría la hipótesis propuesta de que las fibras insolubles a nivel ileal no fermentan, tan solo se solubilizan (Abad-Guamán et al., 2015b), ya que la principal limitante para que la fibra insoluble fermente es el tiempo y a nivel ileal los tiempos de retención son cortos (aproximadamente 3 horas) (García et al., 1999). La fermentación de la fibra soluble a cortos tiempos y de la fibra insoluble a tiempos más largos, hace que la degradación de la fibra dietética total se correlacione con los parámetros de fermentación en todas las horas estudiadas.

Las poca relación de los parámetros de producción de gas in vitro con la degradación cecal de las fibras podría deberse al desbalance de determinación de fermentación de la fibra, ya que a nivel ileal se podría estar solubilizando la fibra y por lo tanto no se recuperan al momento de calcular la digestibilidad, sin necesariamente estar fermentando (Abad-Guamán et al., 2015)., mientras que a nivel cecal es donde realmente se degrada por la rica microbiota y las condiciones óptimas para la fermentación (Gómez-Conde et al., 2007). A nivel fecal la fibra neutro detergente (fracción insoluble de la fibra) es la fracción de la fibra que mejor se relaciona, posiblemente a que en este nivel ha tenido el tiempo suficiente para que su fracción degradable. 


\section{CONCLUSIONES}

En base a los resultados y discusión de cada una de las variables en estudio se pudo determinar que:

La metodología de producción de gas in vitro puede ser utilizada para entender la fermentación de la fibra, en diferentes partes del tracto digestivo in vivo.

El estudio de fermentación de la fibra soluble debe realizarse en la metodología de producción en gas in vitro durante las primeras 6 horas de fermentación.

El estudio de la fermentación de la fibra insoluble (fibra neutro detergente) debe realizarse sobre las 29 horas de fermentación en la metodología de producción de gas in vitro.

\section{FINANCIAMIENTO}

No monetario.

\section{AGRADECIMIENTO}

A la Escuela Superior Politécnica de Chimborazo, Riobamba; por motivar el desarrollo de la Investigación.

\section{REFERENCIAS CONSULTADAS}

Abad-Guamán, R; Carro, M.D; Carabaño, R y García, J. (2015). Estudio de la cinética de producción de gas de la pulpa de remolacha con inóculos ileales y cecales de conejos: Comparación de Modelos [Study of the kinetics of gas production of beet pulp with ileal and cecal inoculums of rabbits: Comparison of Models]. AIDA (2015), XVI Jornadas sobre Producción Animal, Tomo I, 275-277. https://n9.cl/4v32d

Abad-Guamán, R; Carabaño, R; Gómez-Conde, M; García, J. (2015). Effect of type of fiber, site of fermentation, and method of analysis on digestibility of soluble and insoluble fiber in rabbits. Journal of Animal Science, (93), 6, 2860-2871. https://doi.org/10.2527/jas.2014-8767

De Blas C. \& Wiseman J. (2010). The Nutrition of the Rabbit. Cab International. 2nd Eition. Recuperado de https://n9.cl/59lfk 
Dihigo L. (2005). Avance en los estudios de fisiología digestiva del conejo en Cuba con el uso de fuentes de alimentos no tradicionales: Consideraciones Fisiológicas [Advances in rabbit digestive physiology studies in Cuba with the use of nontraditional food sources: Physiological Considerations]. Recuperado de https://n9.cl/lhkiv

García, J., Carabaño, R., \& de Blas, J. C. (1999). Effect of fiber source on cell wall digestibility and rate of passage in rabbits. Journal of animal science, 77(4), 898905. https://doi.org/10.2527/1999.774898x

Gidenne, T., V. Pinheiro, y L. Falcão E Cunha (2000). A Comprehensive Approach of the Rabbit Digestion: Consequences of a reduction in dietary fibre supply. Recuperado en noviembre de 2020, de https://www.researchgate.net/publication/222694474

Gómez-Conde, M. S., García, J., Chamorro, S., Eiras, P., Rebollar, P. G., Pérez de Rozas, A., Badiola, I., de Blas, C., \& Carabaño, R. (2007). Neutral detergent-soluble fiber improves gut barrier function in twenty-five-day-old weaned rabbits. Journal of animal science, 85(12), 3313-3321. https://doi.org/10.2527/jas.2006-777

Kwakkel, R. P., Williams, B. A., \& van der Poel, A. F. B. (1997). Effects of fine- and coarse particle diets on gizzard growth and fermentation characteristics of the caecal contents in broiler chickens. In Proc. 11th European Symp. on Poultry Nutrition (WPSA), Faaborg, Denmark, 24-28 August (pp. 249-251).

Marounek, M., S. Vovk, \& V. Benda. (1997). Fermentation patterns in rabbit caecal cultures supplied with plant polysaccharides and lactate. Acta Veterinaria Brno 66: 9-13. https://doi.org/10.2754/avb199766010009

Menke, K., Raab, L., Salewski, A., Steingass, H., Fritz, D., \& Schneider, W. (1979). The estimation of the digestibility and metabolizable energy content of ruminant feedingstuffs from the gas production when they are incubated with rumen liquor in vitro. The Journal of Agricultural Science, 93(1), 217-222. https://doi.org/10.1017/S0021859600086305

Tilley J M A \& Terry R A. (1963). A two-stage technique for the in vitro digestion of forage crops. J. Brit. Grassland Soc. 18:104-11.

Wilfart, Y. Jaguelin-Peyraud, H. Simmins, J. Noblet, Jaap van Milgen, et al. (2008). Kinetics of enzymatic digestion of feeds as estimated by a stepwise in vitro method. Animal Feed Science and Technology, Elsevier Masson, 141 (1-2), 171183. 
Revista Arbitrada Interdisciplinaria KOINONIA

Año VI. Vol VI. N${ }^{\circ} 1$. Enero - Junio. 2021

Hecho el depósito de Ley: FA2016000010

ISSN: 2542-3088

FUNDACIÓN KOINONIA (F.K). Santa Ana de Coro. Venezuela.

Dalton Euclides Sarango-Guamán; Diego Armando Masaquiza-Moposita; Junior Stalin Vargas-Hidalgo

Wiliams, B. A. (2000). Cumulative gas production techniques for forage evaluation. In D. I. Givens, E. Owen, R. F. E. Axford, \& H. M. Omed (Eds.), Forage Evaluation in Ruminant Nutrition (pp. 189-213). CABI Publishing Wallingford. https://doi.org/10.1079/9780851993447.01

(C2021 por los autores. Este artículo es de acceso abierto y distribuido según los términos y condiciones de la licencia Creative Commons Atribución-NoComercial-Compartirlgual 4.0 Internacional (CC BY-NC-SA 4.0) (https://creativecommons.org/licenses/by-nc-sa/4.0/). 